\title{
Dually actuated atomic force microscope with miniaturized magnetic bead- actuators for single-molecule force measurements
}

Semih Sevim ${ }^{\mathrm{a}}$, Sevil Ozer ${ }^{\mathrm{a}}$, Luying Feng ${ }^{\mathrm{a}}$, Joel Wurzel ${ }^{\mathrm{b}}$, Arielle Fakhraee ${ }^{\mathrm{c}}$, Naveen Shamsudhin $^{\mathrm{d}}$, Bumjin Jang ${ }^{\mathrm{d}}$, Carlos Alcantara ${ }^{\mathrm{d}}$, Olgaç Ergeneman ${ }^{\mathrm{d}}$, Eva Pellicer ${ }^{\mathrm{e}}$, Jordi Sort $^{\mathrm{f}}$, Tessa Lühmann ${ }^{\mathrm{b}}$, Salvador Panéd ${ }^{\mathrm{d}}$, Bradley J. Nelson ${ }^{\mathrm{d}}$, Hamdi Torun, ${ }^{\mathrm{a}^{*}}$

${ }^{a}$ Department of Electrical and Electronics Engineering, Bogazici University, Bebek 34342 Istanbul, Turkey

${ }^{b}$ Institute of Pharmacy and Food Chemistry, University of Würzburg, Würzburg, Germany

${ }^{c}$ Aeon Scientific AG, Schlieren, 8952, Zurich, Switzerland

${ }^{d}$ Multi Scale Robotics Lab, Institute of Robotics and Intelligent Systems, ETH Zurich, 8092, Zurich, Switzerland

e Departament de Física, Facultat de Ciències, Universitat Autònoma de Barcelona, E-08193 Bellaterra, Spain.

${ }^{f}$ Institució Catalana de Recerca i Estudis Avançats (ICREA) and Departament de Física, Universitat Autònoma de Barcelona, E08193 Bellaterra, Spain.

${ }^{*}$ Corresponding author. E-mail address: hamdi.torun@boun.edu.tr 


\begin{abstract}
We report for the first time on a novel Atomic Force Microscopy (AFM) technique with dual actuation capabilities using both piezo and magnetic bead actuation for advanced single-molecule force spectroscopy experiments. The experiments are performed by manipulating functionalized magnetic microbeads using an electromagnet against a stationary AFM cantilever. Magnetic actuation has been demonstrated for AFM before to actuate cantilevers, but here we report for the first time a method where we keep the cantilever stationary and accomplish actuation via free-manipulated microstructures. This method leads to a significant reduction of mechanical drift in the system since the experiments are performed without a need for a hard surface and the measured force between the cantilever and the bead is inherently differential. In addition, shrinking the size of the actuator can minimize hydrodynamic forces affecting the AFM cantilever. We conducted single-molecule force spectroscopy and force-clamp experiments with biotin/streptavidin as a model system using the new method. The new method reported herein allows applying constant force on the beads to perform forceclamp experiments without any active feedback, which might be crucial for a deeper understanding of interaction between biomolecules.
\end{abstract}

Keywords: atomic force microscopy; magnetic beads; force spectroscopy; singlemolecule experiments 
Atomic force microscopy (AFM) has been used for three decades in the field of molecular biology and molecular biophysics as a versatile force measurement tool. AFM offers a force resolution of a few pico-Newtons in liquid environments at the single-molecule level [1]. The potential of the technology has been demonstrated successfully for various biomolecular measurements, such as antibody-antigen binding, receptor-ligand interaction, and protein folding/unfolding measurements at single-molecule levels using the biomolecular force spectroscopy technique [2-5]. Basically, the force spectroscopy technique involves a cantilever that is functionalized with a specific type of biomolecules. The motion of the cantilever is controlled conventionally using a piezo-electric actuator over a sample surface that can also be functionalized with biomolecules. The actuator brings the cantilever in and out of contact with the sample surface repeatedly while the force on the cantilever is detected by optical means. The technique allows the investigation of the specific interactions between single pairs of biomolecules located at the functionalized tip and the sample surface in aqueous environments. It is feasible to study quantitatively the structure, function and energy landscape of biomolecular interactions. The cantilever is actuated with a wide range of speeds to load the biological bond before it breaks while the corresponding forces of biomolecular interactions are obtained. Thus, it is advantageous to have both stable actuators with minimized drift to enable low speed actuation and fast actuators to enable high-speed actuation. One of the main limitations for the actuation is the large piezoactuation of the cantilever through the holders. This may introduce additional modes into the complete mechanical system, and also limits the actuation capabilities at high speeds measurements [6]. In addition to large piezo actuation, cantilever geometry also has a direct role on the limitation of the actuation speed due to the hydrodynamic drag effects $[7,8]$. Many researchers have proposed different methods, such as magnetic actuation [9], photothermal actuation [10], acoustic pressure actuation [11] and capacitive actuation [12] to actuate the cantilevers directly. Magnetic cantilevers [13] or conventional cantilevers attached with magnetic particles [14] can be actuated by coupling the AFM head with an electromagnet or a permanent magnet.

Drift in the system negatively affects the measurements, especially at low actuation speeds by inducing spurious deflections on the cantilever. The basic sources of drift in the system are 
mechanical instabilities and thermally induced deflection of the cantilever. Deflection of the cantilever towards the substrate increases the contact forces on the biomolecules and eventually can harm the biological specimens $[15,16]$. In addition, deflection of the cantilever due to drift results in false force readings. Several methods have been presented to overcome the effects of thermal drift problem in AFM using modified cantilevers, new probes [17-19], reference cantilevers [20,21] and thermomechanically compensating microstages [15].

In this study, we present a novel AFM technique with dual actuation capability. The AFM employs a conventional piezotube actuator together with a new approach where we use magnetic beads as a secondary actuator. Using magnetic beads minimizes the size of the actuator down to a size comparable to that of a single molecule. In this architecture, a molecule attached to an AFM cantilever is probed with a counter interacting molecule attached to a magnetic bead. The cantilever is kept stationary and is used as a force sensor. Under the externally controlled magnetic field and field gradient, it is possible to manipulate the magnetic bead without a substrate surface. Magnetic actuation has been demonstrated for AFM before to actuate cantilevers [13,14], but we report for the first time a method where the cantilever is kept stationary and the actuation is accomplished via freemanipulated microstructures. Manipulation tasks with objects varying in dimensions from nanometres to centimetres with five degrees-of-freedom with high resolution that is limited by imaging technology have already been demonstrated with electromagnetic manipulation setups [22]. The footprint of the magnetic actuator with a diameter of a few microns is orders of magnitude smaller than the commonly used piezotubes. Use of miniaturized mechanical actuator improves system dynamics since the cantilever is kept stationary and a dynamic magnetic bead encounters drastically smaller hydrodynamic drag forces in viscous fluids as compared to larger structures. The drag (hence damping) on a mechanical system is an indication of thermal noise according to the fluctuation dissipation theorem. Reduced hydrodynamic drag improves force resolution and the stability of the system significantly. 


\section{Results}

\section{Characterization}

The AFM system including an integrated electromagnet and a magnetic bead-based actuator is shown in Figure 1. The AFM head includes a piezoactuator and the space below the cantilever plane is kept empty so that the AFM head can be integrated with a magnetic manipulator. An electromagnet including a core and coil is depicted in Figure 1 to actuate a magnetic particle in one-dimensional translational motion for biomolecular pulling measurements.

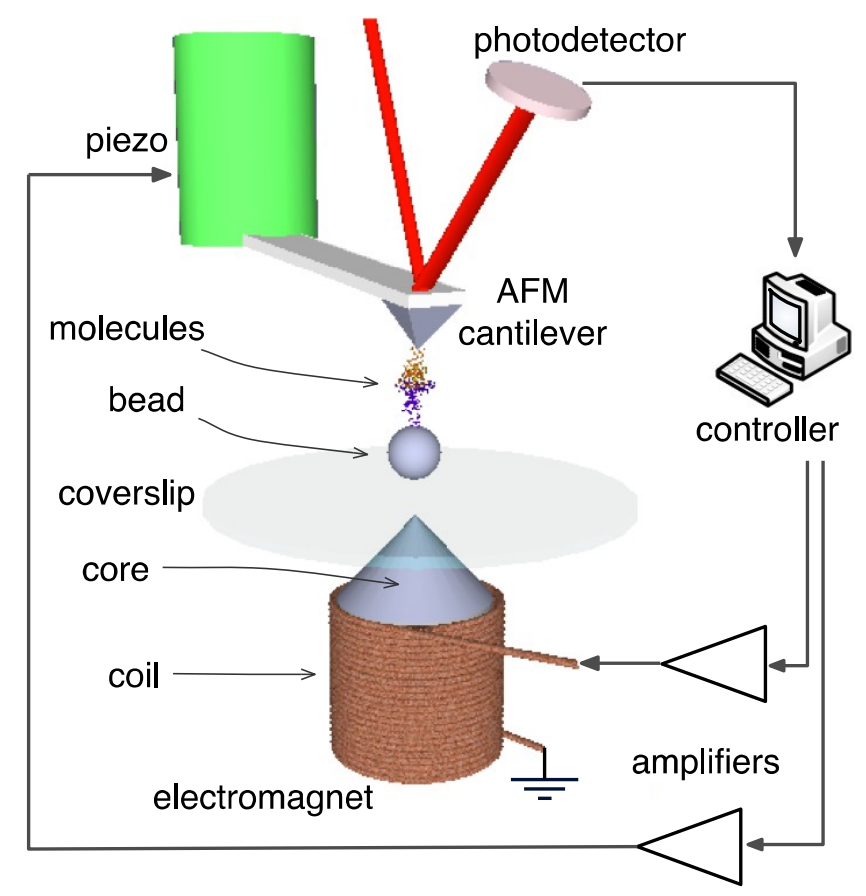

Figure 1. Schematic overview of the AFM system with dual actuators.

In a typical force spectroscopy experiment, the magnetic bead is actuated against the cantilever to probe the interactions between the molecules attached to the bead and the cantilever. The experiment using the piezoactuation of the cantilever and magnetic actuation of the magnetic bead is described schematically in Figure 2. The cantilever is functionalized with molecule A and tested against to molecule B attached to the magnetic beads in aqueous environment at room temperature. First, the functionalized cantilever is brought down using the piezoactuator to the substrate, which is covered with magnetic beads (0). The cantilever is first pressed into the substrate to allow molecules to form 
bonds. Then, the cantilever is pulled away from the substrate using the piezoactuator (I). As the cantilever goes out of contact with the substrate, non-specific adhesions can be observed (II). Finally, the cantilever goes completely out of contact and then the piezoactuator stops (III).

Piezo Actuation

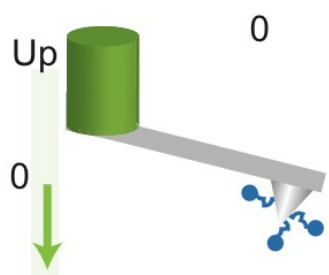

Down

$\underline{U} \quad$ Up
Up
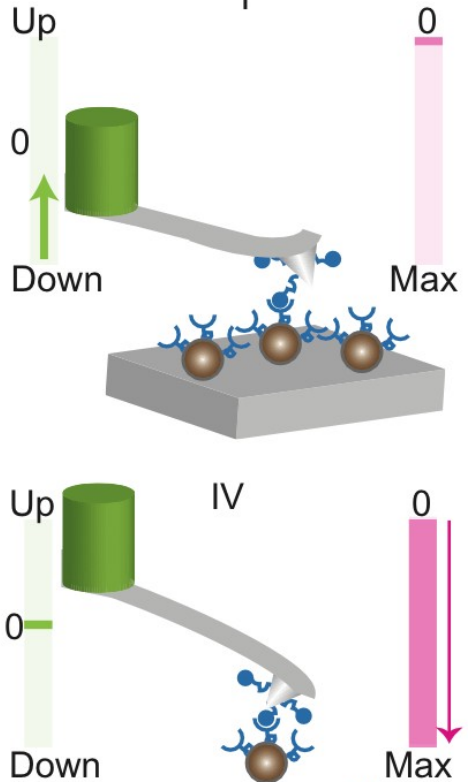

$\operatorname{Max}$

Down
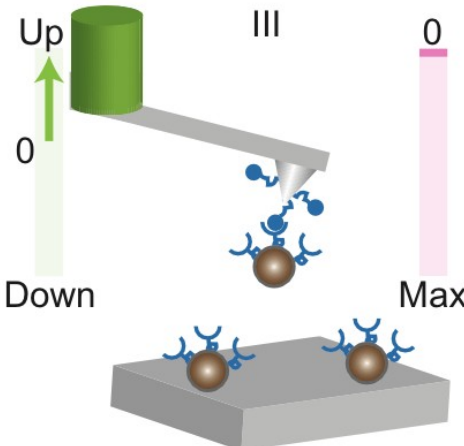

Down

\section{Magnetic Actuation}

II
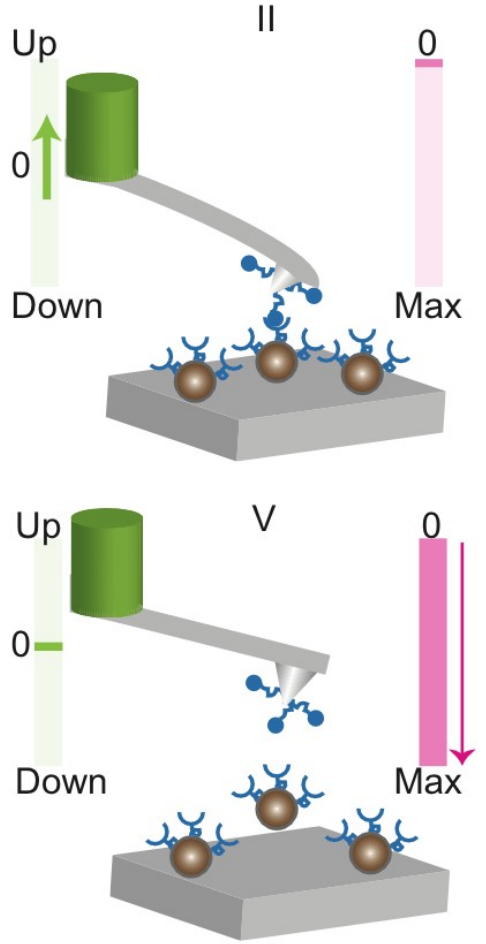

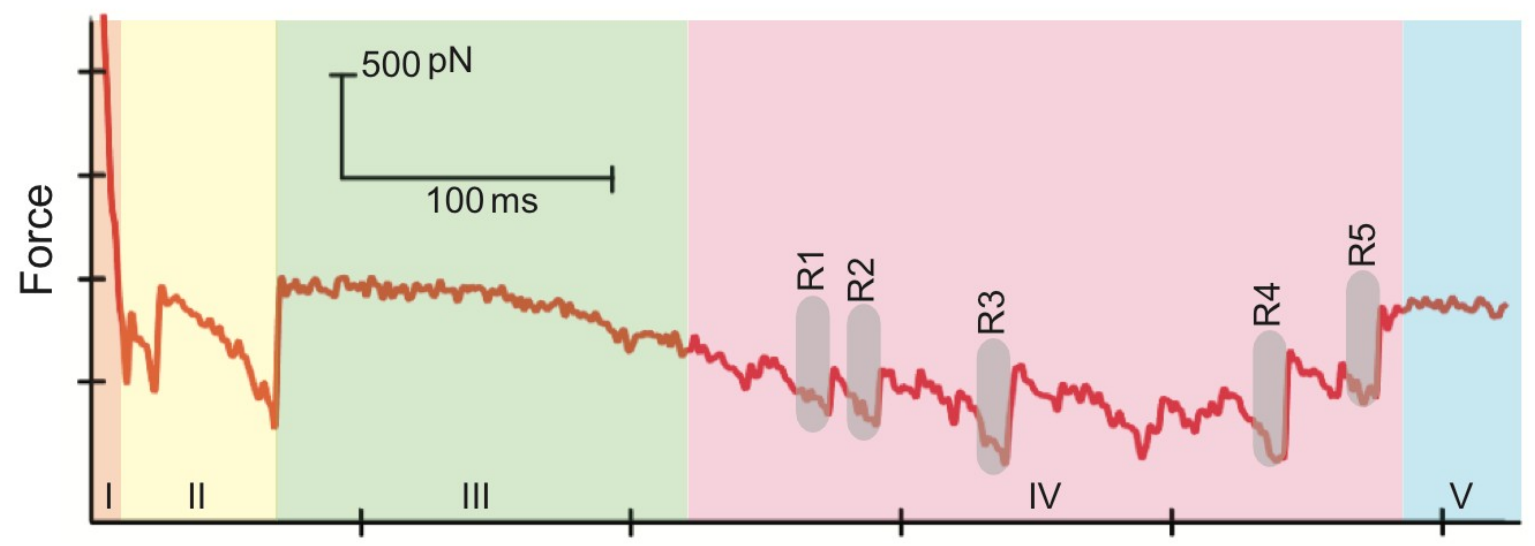

Time

Figure 2. Schematic representation of the dual actuation capability of the system $(0-\mathrm{V})$. The arrows indicate the direction of actuation using the piezo- and magnetic actuator. A sample force curve 
obtained during a biomolecular experiment is shown with labels corresponding to different phases (0V). Multiple single molecular events (R1-R5) during phase IV are resolved clearly.

After the piezoactuator is turned off, the electromagnet is driven to pull the magnetic beads back to the substrate (IV). Electromagnetic actuation may lead to unbinding of the molecules located at the cantilever and the magnetic beads, showing molecular rupture events. (IV). Multiple rupture events labeled as R1-R5 are observed in region IV while the coil is on. The unbinding forces of the rupture events are ranging from 100 to $500 \mathrm{pN}$ on the specific force curve. The last phase (V) on the force curve indicates all the beads are detached from the cantilever.

The number of events in one approach cycle can be controlled with the numbers of beads located on the substrate. The time and force resolution of the system allows identifying single events and measuring both the loading rate of the molecular bond and the unbinding force. A typical force time trace showing an unbinding event with a force of $50 \mathrm{pN}$ at a loading rate of $70 \mathrm{pN} / \mathrm{s}$ is depicted in Figure 3. The demonstrated loading rate is significantly low, but the system allows the accurate measurement of force curves at low loading rates and low force levels.

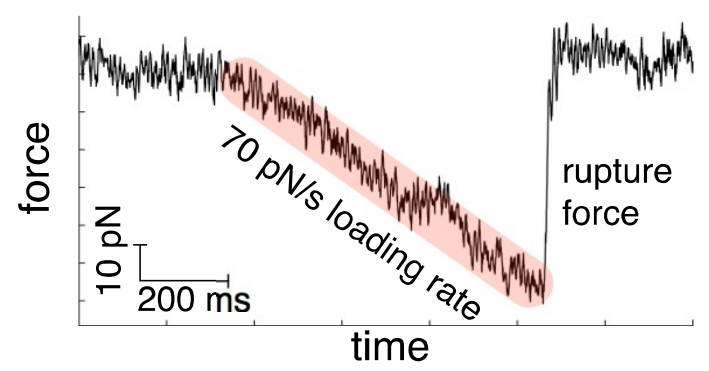

Figure 3. A sample force curve with $70 \mathrm{pN} / \mathrm{s}$ loading rate and showing $50 \mathrm{pN}$ unbinding force.

The method of magnetic actuation eliminates the need for the interaction between the cantilever and a sample surface while measuring the unbinding events. The capability of performing experiments away from the sample surface is advantageous since the large mechanical loop between the cantilever and 
the sample surface is broken. In a typical AFM setup, the cantilever and the sample surface are located on different planes and the stability of the relative position of the planes are determined by the rigidity of all the mechanical components in the mechanical loop connecting these planes. The mechanical loop is a significant source of drift in conventional AFM setups [20,23]. In addition, keeping the cantilever away from the sample surface improves its dynamics by eliminating the hydrodynamic wall effect $[13,24]$. We performed long-timescale drift experiments using our new AFM system in comparison with a commercial AFM system (Dimension Edge, Bruker). We actuated an AFM cantilever (MLCT-C type, V shaped Silicon Nitride cantilever, Bruker) in and out-off contact with a coverslip using the piezoactuator of the commercial system to characterize drift in the setup. For this purpose, we applied a square wave signal with constant amplitude to the piezo and monitored the deflection of the cantilever. Figure 4 (black line) shows the force on the cantilever. The detected signal follows a square wave with increasing amplitude. The increase in the force amplitude is an indication of the drift in the system as the position of the cantilever changes relative to the coverslip in time. The amplitude increases by a factor of 2.5 in $500 \mathrm{~s}$ for the specific experiment. Apparently, the distance between the base of the cantilever and the sample surface decreased due to the drift in the system. This resulted in an increase in the contact force. Increase in the contact force is detrimental especially when the tip of the cantilever is functionalized with biomolecules as they can be harmed. Simultaneously, we conducted a similar experiment with our new AFM setup. We glued a ferromagnetic bead with a diameter of $30 \mu \mathrm{m}$ on an identical cantilever and actuated the beadattached cantilever by applying a square wave signal with constant amplitude to the electromagnet while observing the deflection. We performed both the experiments at the same time to have identical effect due to environmental disturbance. The red line in Figure 4 shows the deflection of the cantilever due to the magnetic actuation. The amplitude of deflection signal keeps constant during the entire experiment. The mean value of the deflection signal decreases with respect to time due to the temperature response of the cantilever. On the other hand, the differential amplitude of the force on the cantilever remains the same. Measurements of biomolecular forces rely on the differential force, so the method of actuation minimizes the detrimental effects of drift. Also, the biomolecules do not 
experience excessive force due to the thermally induced forces on cantilever since a rigid mechanical loop between the cantilever and the substrate is eliminated.

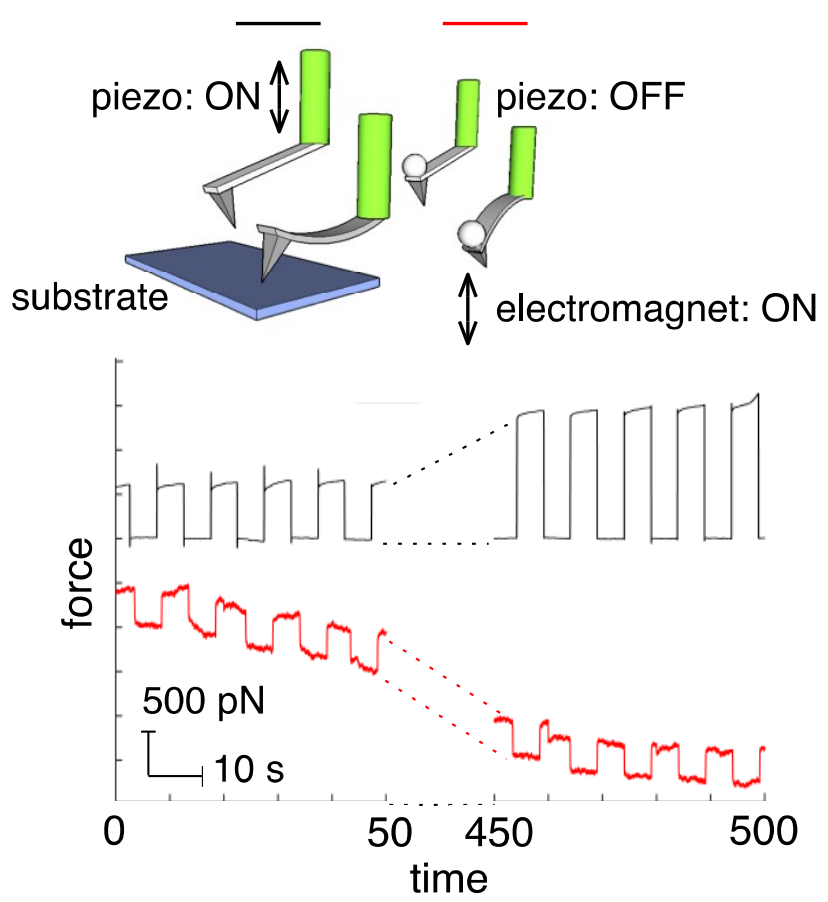

Figure 4. Time traces for the characterization of drift obtained using a commercial AFM system (upper, black trace) and magnetic actuation of a bead-attached cantilever using custom-built AFM setup (lower, red trace).

Another inherent advantage of the magnetic bead actuation method is the capability of performing force clamp experiments without a need for active feedback. The current applied to the electromagnet determines the strength of the magnetic field that determined the force applied to the beads. So, the force applied on magnetic beads is constant when the electromagnet is driven with a constant current signal. Right after the functionalized cantilever grabs a magnetic bead (see Figure 2-III), force-clamp experiments can be performed by applying a constant force on the bead until an unbinding event is observed. The duration of time between the instance when the force is applied and the instance of unbinding, i.e. lifetime, is recorded. A typical force time trace for a force-clamp experiment is shown in Figure 5 without any feedback. We set a clamping force of $50 \mathrm{pN}$ between the molecules and measured a life-time of 600 ms. 


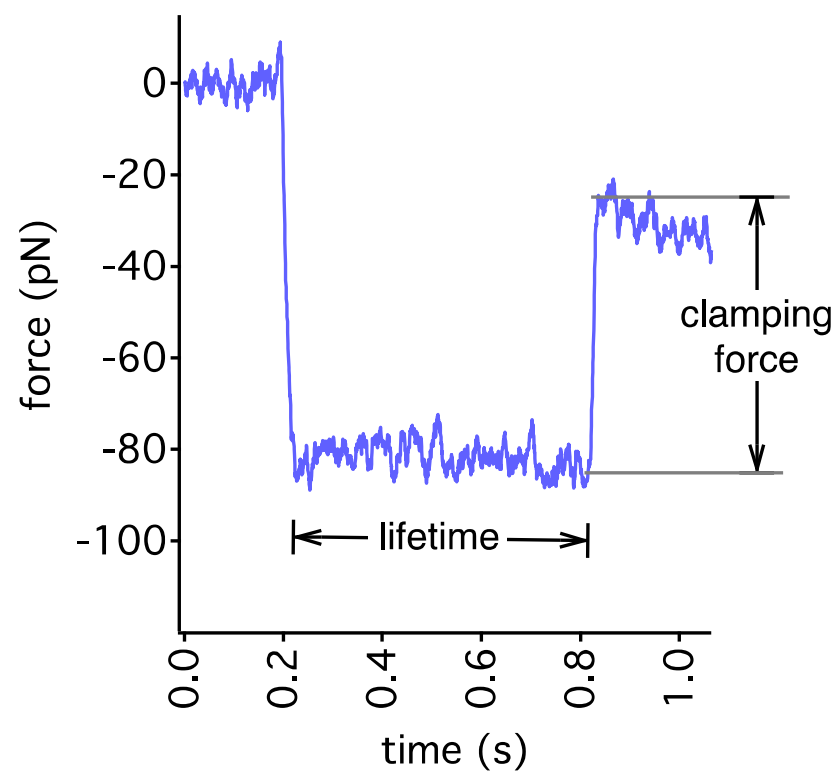

Figure 5. A sample force time trace in a force-clamp setting by applying constant current to the magnetic manipulator without any feedback loop requirements.

In a conventional AFM setup, the measurement of lifetimes is possible using an active feedback control to keep the force between the molecules constant. The drift in the setup requires accurate control of the piezoactuator. Figure 6 shows a typical force time trace together with the piezo displacement in a force-clamp setting. We used a biotinylated cantilever and a cover slip incubated with streptavidin for the demonstration experiment. We set the clamping force to $200 \mathrm{pN}$ between the molecules and adjusted the controller settings for the piezoactuator of the cantilever. The displacement data shows clearly the piezo was actuated $>30 \mathrm{~nm}$ to keep the force constant during the clamping period. In addition, the closed-loop feedback control usually reduces the detection bandwidth. On the contrary, the method of magnetic bead actuation does not need an active feedback control for force-clamp experiments and offers improved force-clamp measurements. 

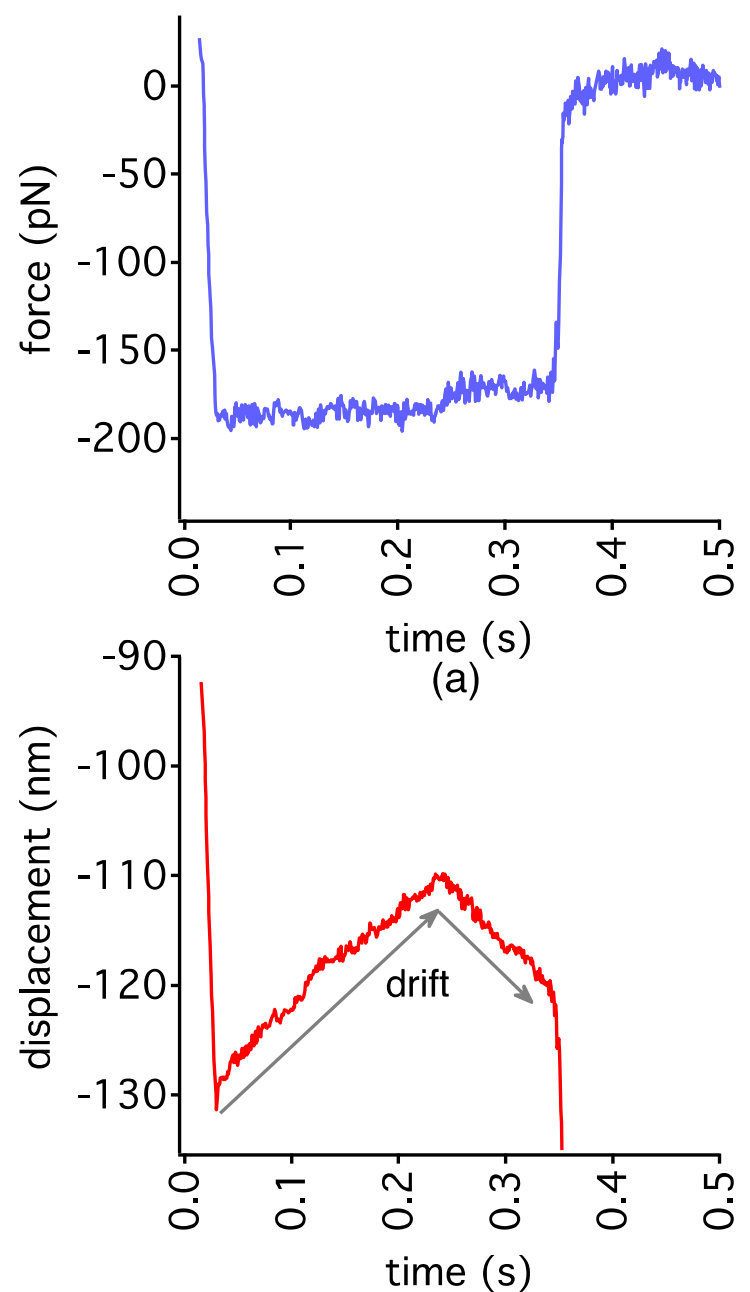

(b)

Figure 6. Force-clamp measurements using piezoactuator of a commercial AFM system with active feedback to control the displacement of the piezoactuator to keep the force between the molecules the constant.

\section{Biomolecular Experiment}

We performed a biomolecular experiment using the dual actuation capability of our AFM setup. We used a biotin-coated cantilever for this experiment and tested it against streptavidin functionalized magnetic beads with a diameter of $2.8 \mu \mathrm{m}$. We followed the protocol described in Figure 2 at room temperature during the experiment. First, we actuated the cantilever using the piezo towards a hard surface where the beads were located. Then, we retracted the cantilever away from the surface and turned off the piezo. Finally, we turned on the magnetic actuator to observe unbinding events if the 
cantilever had picked up beads in the approach cycle. We collected several hundreds of force curves at different loading rates. The force histograms are shown in Figure 7(a). We recorded the probability of specific events less than 30\%. Figure 7(b) shows the unbinding forces as a function of loading rates. The measured values of the unbinding forces between biotin and streptavidin are comparable to the results published in literature. We also performed control experiments by saturating biotin on the cantilever with excess streptavidin. The addition of excess streptavidin on the cantilever reduces the probability of the specific events down to $1 \%$, validating the experimental protocol. 


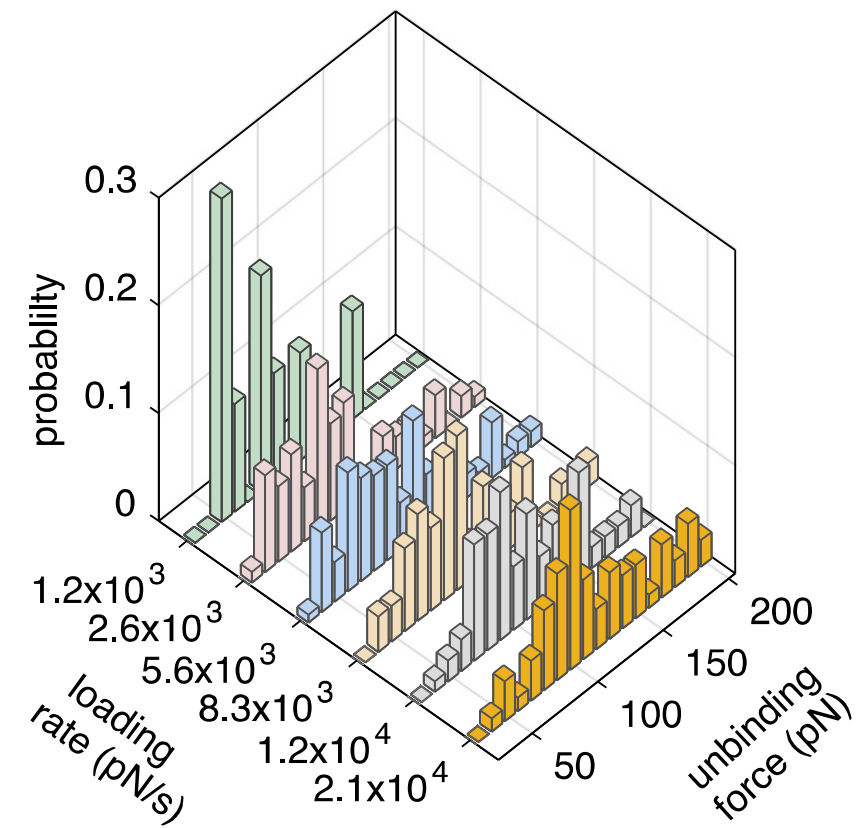

(a)

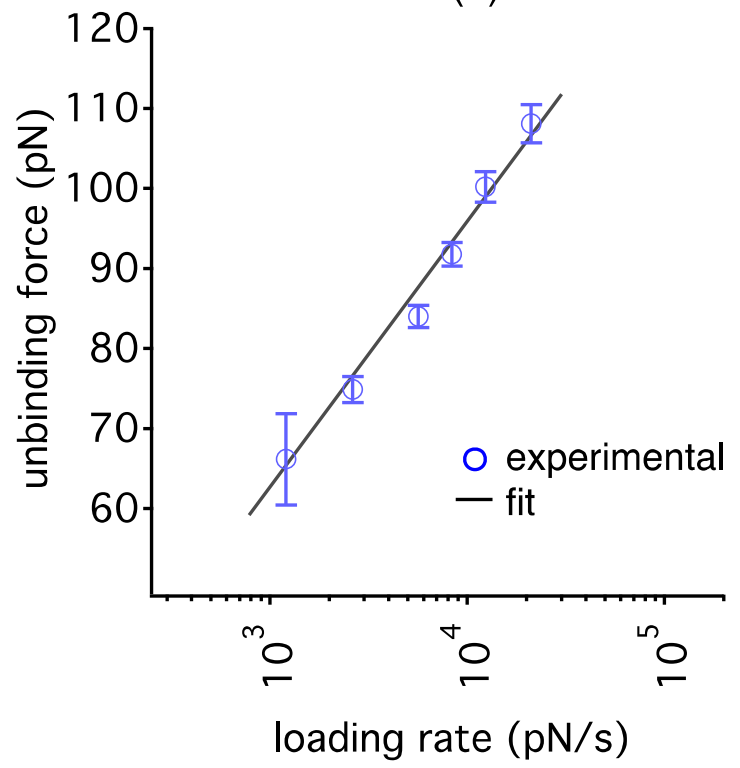

(b)

Figure 7. (a) Force histograms for the unbinding force levels of single biotin-streptavidin pairs obtained at different loading rates. (b) Unbinding force vs. loading rates graph for biotin/streptavidin pulling experiments using the method of magnetic bead actuation

\section{Discussion}

We have developed a novel AFM technique with a new AFM setup for single-molecule force spectroscopy experiments to meet current limitations. The AFM system has dual actuation capabilities using a conventional piezoactuator and a magnetic actuator based on commercial available and 
surface functionalized magnetic beads. The piezo of the AFM system is mainly used to position the AFM cantilever and is turned off during the data acquisition phase. Instead of actuating the cantilever, a magnetic bead is manipulated against the cantilever by an electromagnet comprising a coil and a core in the demonstrated novel AFM technique. The beads that we use are $2.8 \mu \mathrm{m}$ in diameter. The advantages of such a small mechanical actuator are numerous in terms of dynamics, resolution and stability. Using a free-manipulated micron-sized bead minimizes the effects of drift and hydrodynamic forces on the force-sensing cantilever. In addition, the force spectroscopy can be performed away from rigid surfaces, which might contribute to denaturation of the biomolecule thus providing artefacts during force spectroscopy analysis. The introduced method combines the technological advancements of AFM and magnetic tweezers. The force measurement exploits the versatility of AFM as it is performed using a stationary cantilever. On the other hand, the actuation relies on the manipulation of a micron-sized bead that benefits from the stability and resolution of magnetic beads. The size of the actuator beads can potentially be reduced down to the sizes comparable with single molecules. Here we have demonstrated for the first time the concept of the new method and the new instrument. The size of the actuator is minimized and the large mechanical loop between the cantilever and any rigid substrate is broken. Hence, using the magnetic actuation method, low loading rates down to $10^{1} \mathrm{pN} / \mathrm{s}$ with single-molecules were demonstrated. We obtained the dynamic strength spectrum between biotin and streptavidin using the novel AFM technique and the results were in a good agreement with the conventional piezo actuation methods. In addition, the ability to apply a constant force on the magnetic bead leads to perform force-clamp experiments without the need for an active feedback control. In summary here we combined an AFM system with a magnetic actuator. The new instrument opens exciting new opportunities to study biomolecular interactions in the range of low loading rates at physiological relevant forces.

\section{Methods}

\section{Setup}

Our design includes a custom built AFM head integrated with an electromagnetic manipulator as shown in Figure 1. The cantilever is attached to a piezoactuator (PI-841.1 with a vertical range of 15 
$\mu \mathrm{m}$, Physik Instrumente $\mathrm{GmbH}$ ) and its deflection is monitored using a quadrature photodetector (Pacific Silicon Sensor, CA, USA). The cantilever is illuminated with a fibre pigtailed laser (Oz Optics, Ottowa, Canada).

The electromagnet consists of an optimized ferromagnetic core geometry and copper winding. The core has a sharp tip to localize the magnetic field at a working distance of 100-150 $\mu \mathrm{m}$ from the tip. The exchangeable core-piece is made of FeCo alloy (Vacoflux $50^{\mathrm{TM}}$ ) because of its high magnetization saturation $\left(\mathrm{M}_{\mathrm{s}}=2.35 \mathrm{~T}\right)$, which is capable of generating a magnetic field of $0.55 \mathrm{~T}$ at a working distance of $100 \mu \mathrm{m}$ from the tip for a current density of $4 \mathrm{~A} / \mathrm{mm}^{2}$.

Since biomolecular experiments have strict requirements on temperature control and stability, we placed the electromagnet in a temperature stabilizer unit comprising a thermoelectric cooler (UEPT440-127-079E120, Uwe Electronic $\mathrm{GmbH}$ ) for fine-regulation of temperature on top of a water-based cooling system. Thermoelectric cooler holds the coverslips during the experiments.

We implemented a software-based controller (NI PXI-8102, National Instruments, TX, USA) via an expansion card with 8 analog inputs $\left(F_{s}=1.25 \mathrm{MS} / \mathrm{s}\right), 2$ analog outputs $\left(F_{\mathrm{s}}=3.33 \mathrm{MS} / \mathrm{s}\right)$ and 24 digital inputs/outputs. We programmed the controller and the user interface in NI-LabView environment.

\section{AFM measurements}

We used commercially available biotin-coated cantilevers (CT.BIO, Novascan, Ames IA USA) for the experiments. We calibrated the spring constant and the sensitivity of the cantilevers before and after the experiments. We tested these cantilevers against streptavidin functionalized magnetic beads with a diameter of $2.8 \mu \mathrm{m}$ (Dynabeads, M-280 Streptavidin, ThermoFisher Scientific). The provider company functionalizes the beads with streptavidin and the concentration of the bead suspension is 10 
$\mathrm{mg} / \mathrm{mL}$. We diluted the original suspension by mixing $1 \mu \mathrm{L}$ suspension with $1 \mathrm{~mL}$ Phosphate-buffer saline (PBS). Finally, we performed the experiment in $100 \mu \mathrm{L}$ PBS by adding $5 \mu \mathrm{L}$ diluted bead suspension. 


\section{References}

[1] K.C. Neumanand, and A. Nagy, Single-molecule forcespectroscopy:Optical tweezers, magnetic tweezers and atomic force microscopy, Nature Methods, 5, (2008) 491-505.

[2] E.M. Puchner, and H.E. Gaub, Force and function: probing proteins with AFM-based force spectroscopy, Current Opinion in Structural Biology. 19 (2009) 605-614.

[3] D.J. Muller, and Y.F. Dufrene, Atomic force microscopy as a multifunctional molecular toolbox in nanobiotechnology, Nature Nanotechnology. 3 (2008) 261-269.

[4] I. Popa, P. Kosuri, J. Alegre-Cebollada, S. Garcia-Manyes, and J.M. Fernandez, Force dependency of biochemical reactions measured by single-molecule force-clamp spectroscopy, Nature Protocols. 8 (2013) 1261-1276.

[5] Z.N. Scholl, Q. Li, and P.E. Marszalek, Single molecule mechanical manipulation for studying biological properties of proteins, DNA, and sugars, Wiley Interdisciplinary Reviews- Nanomedicine and Nanobiotechnology. 6 (2014) 211-229.

[6] C. Carrasco, P. Ares, P.J. de Pablo, and J. Gómez-Herrero, Cutting down the forest of peaks in acoustic dynamic atomic force microscopy in liquid, Review of Scientific Instruments. 79 (2008) 126106.

[7] H. Janovjak, J. Struckmeier, and D. Müller, Hydrodynamic effects in fast AFM single-molecule force measurements, European Biophysics Journal. 34 (2005) 91-96.

[8] K. Sarangapani, H. Torun, O. Finkler, C. Zhu, and L. Degertekin, Membrane-based actuation for high-speed single molecule force spectroscopy studies using AFM, European Biophysics Journal. 39 (2010) 1219-1227

[9] C. Vančura, J. Lichtenberg, A. Hierlemann, and F. Josse, Characterization of magnetically actuated resonant cantilevers in viscous fluids, Applied Physics Letters. 87 (2005) 162510.

[10] S.W. Stahl, E.M. Puchner, and H.E. Gaub, Photothermal cantilever actuation for fast singlemolecule force spectroscopy, Review of Scientific Instruments. 80 (2009) 073702. 
[11] F.L. Degertekin, B. Hadimioglu, T. Sulchek, and C.F. Quate, Actuation and characterization of atomic force microscope cantilevers in fluids by acoustic radiation pressure, Applied Physics Letters. 78 (2001) 1628-1630.

[12] J. Zhang, D.M. Czajkowsky, Y. Shen, J. Sun, C. Fan, J. Hu, and Z. Shao, Bias controlled capacitive driven cantilever oscillation for high resolution dynamic force microscopy, Applied Physics Letters. 102 (2013) 073110.

[13] C. Rankl, V. Pastushenko, F. Kienberger, C.M. Stroh, and P. Hinterdorfer, Hydrodynamic damping of a magnetically oscillated cantilever close to a surface, Ultramicroscopy. 100 (2004) 301308.

[14] G.R. Jayanth, Y. Jeong, and C.-H. Menq, Direct tip-position control using magnetic actuation for achieving fast scanning in tapping mode atomic force microscopy, Review of Scientific Instruments. 77 (2006) 053704.

[15] S. Sevim, S. Tolunay, and H. Torun, Micromachined sample stages to reduce thermal drift in atomic force microscopy, Microsystem Technologies. 21 (2014) 1559-1566.

[16] H. Torun, O. Finkler, and F.L. Degertekin, Athermalization in atomic force microscope based force spectroscopy using matched microstructure coupling, Review of Scientific Instruments. 80 (2009) 076103.

[17] A. Beyder, C. Spagnoli, and F. Sachs, Reducing probe dependent drift in atomic force microscope with symmetrically supported torsion levers, Review of Scientific Instruments. 77 (2006) 056103056105.

[18] P.P. Weafer, J.P. McGarry, M.H. van Es, J. Kilpatrick, W. Ronan, D.R. Nolan, and S.P. Jarvis, Stability enhancement of an atomic force microscope for long-term force measurement including cantilever modification for whole cell deformation, Review of Scientific Instruments. 83 (2006) 093709-093710.

[19] L. Wenzler, G. Moyes, and T. Beebe, Improvements to atomic force microscopy cantilevers for increased stability, Review of Scientific Instruments. 67 (1996) 4191-4197.

[20] S.M. Altmann, P.F. Lenne, and J.K. Heinrich Horber, Multiple sensor stabilization system for local probe microscopes. Review of Scientific Instruments. 72 (1996) 142-149. 
[21] J.L. Choy, S.H. Parekh, O. Chaudhuri, A.P. Liu, C. Bustamente, M.J. Footer, J.A. Theriot, and D.A. Fletcher, Differential force microscope for long time-scale biophysical measurements. Review of Scientific Instruments, 78 (2007) 043711-043716.

[22] J. Pokki, O. Ergeneman, S. Sevim, V. Enzmann, H. Torun, and B.J. Nelson, Measuring localized viscoelasticity of the vitreous body using intraocular microprobes. Biomedical Microdevices, 17 (2015) 1-9.

[23] J.H. Kindt, J.B. Thompson, M.B. Viani, and P.K. Hansma. Atomic force microscope detector drift compensation by correlation of similar traces acquired at different setpoints. Review of Scientific Instruments, 73 (2002) 2305.

[24] E. Bonaccurso, M. Kappl, and H.-J. Butt. Hydrodynamic Force Measurements: Boundary Slip of Water on Hydrophilic Surfaces and Electrokinetic Effects. Physical Review Letters, 88 (2002) 076103.

\section{Acknowledgments}

Authors would like to acknowledge funding from the EC (ICT FET-Open) under the MANAQA Project, Grant no 296679.

\section{Author contributions}

SS and SO designed the AFM head. AF, NS, BJ and CA designed the magnetic manipulator. JW and TL performed the bio-functionalization, SS, SO and LF conducted the experiments. SS, SO and HT wrote the main manuscript text. EP and JS characterized magnetic particles, OE, EP, JS, TL, SP, BJN and HT planned and organized the project. All authors reviewed the manuscript.

\section{Competing financial interests}

The author(s) declare no competing financial interests. 